\title{
Nonasymptotic analysis of relativistic electron scattering in the Coulomb field
}

\author{
I. D. Feranchuk and O. D. Skoromnik \\ Belarusian State University, 4 Nezavisimosty Avenue, BY-220030 Minsk, Belarus
}

(Received 18 May 2010; published 9 November 2010)

\begin{abstract}
It is shown that the conventional Born series for relativistic electron scattering in the Coulomb field cannot be used for calculating the scattering characteristics. The differential cross section at small scattering angles is found on the basis of the Furry-Sommerfeld-Maue solution of the Dirac equation. Propagation of the electron wave packet is considered in order to separate the incident and scattered fluxes. It is shown that the total scattering cross section proves to be finite but depends on the distance $r$ between the scattering center and the observation point. It is also shown that the polarization characteristics of the scattered beam are changed due to the long-range character of the Coulomb potential. The results can be important because Coulomb scattering is often used for normalization of experimental data in high-energy physics.
\end{abstract}

DOI: $10.1103 /$ PhysRevA.82.052703

PACS number(s): 03.65.Nk, 11.80.Fv

\section{INTRODUCTION}

The problem of scattering of a charged particle in the Coulomb field is described in textbooks on quantum mechanics and quantum electrodynamics $[1,2]$. It is the generally accepted view that the differential cross section $d \sigma(\theta)$ of the particle scattered in the solid angle $d \Omega$ is described by the Rutherford formula in the nonrelativistic case $(\hbar=c=1)$,

$$
\frac{d \sigma}{d \Omega}=\left(\frac{Z \alpha}{2 m v^{2}}\right)^{2} \frac{1}{\sin ^{4} \frac{\theta}{2}},
$$

and the Mott formula for the relativistic particle,

$$
\frac{d \sigma}{d \Omega}=\left(\frac{Z \alpha}{2 p v}\right)^{2}\left(1-v^{2} \sin ^{2} \frac{\theta}{2}\right) \frac{1}{\sin ^{4} \frac{\theta}{2}},
$$

where $Z$ is the nucleus charge number; $m, p$, and $\varepsilon$ are the particle mass, momentum, and energy, respectively; $v=\varepsilon / p$; $\alpha=\frac{e^{2}}{4 \pi} ;$ and $\theta$ is the scattering angle.

Thus, the main measurable characteristic of the scattering process has a nonintegrable singularity as $\theta \rightarrow 0$. One may argue that this singularity does not lead to any problem because the detector cannot be arranged at such a small angular range in a real experiment. Besides, the Coulomb potential is usually screened in real matter and the scattering amplitude is finite for all angles in this case. However, there are a series of reasons for investigating this singularity further. First, this singularity means that the flux of scattered particles could exceed the incident flux and this is a violation of the particle conservation law. This fact contradicts the finite value of the exact wave function of the system in the whole space. Second, there are some physical characteristics (for example, mobility of charged particles in semiconductors or parameters of the kinetic equations in plasma) that depend on the total or transport cross sections:

$$
\sigma_{\mathrm{tot}}=\int d \sigma(\theta), \quad \sigma_{\mathrm{tr}}=\int(1-\cos \theta) d \sigma(\theta),
$$

and some phenomenological parameters should be introduced in order to calculate them in Refs. [3-5].

This problem was analyzed in detail in a recent paper [6] for the nonrelativistic case. It was shown that in the case of the Coulomb potential the conventional asymptotic form of the wave function was unavailable for the calculation of the differential cross section in the range of small scattering angles. Fortunately, there is an exact solution of the Schrödinger equation with the Coulomb potential. Then the correct flux of the scattered particles was calculated and the differential cross section was defined. It proved to be finite in the entire angular range. The total cross section and the transport cross section were also finite and dependent on the distance between the scattering center and the position of the detector. The conservation of particle flux was proved. It could be considered an analog of the "optical" theorem in the case of the Coulomb scattering.

It is of great interest to generalize these results for relativistic particles, namely for experiments in high-energy physics when electrons collide with bare nuclei without screening. In this case, the specific effects of the long-range Coulomb potential could be essential for analysis of the experimental data. In accordance with the results of Refs. [6,7], the conventional Born series is unavailable for the description of these effects and the accurate nonasymptotic wave function should be used in this case. Unlike the nonrelativistic case, the analytical exact solution of the Dirac equation in the Coulomb field is known only in the spherical coordinate system that is inconvenient for the small-angle scattering problem. Therefore, the Furry-Sommerfeld-Maue (FSM) approximation [8] for the wave function in the parabolic coordinates is used in our paper for nonasymptotic analysis of the scattering problem at small angles. It allows us to show that all observable values are finite in a whole angular range and can be calculated in a closed form. The propagation of the scattered electron wave packet is analyzed in order to divide the incident and scattered fluxes in the case of small scattering angles when the wave function has no conventional asymptotic form. It is also shown that the different polarizations of the incident and scattered particles appear in the relativistic case. Hence, the measurement of polarization may allow one to separate the scattered particle flux from the incident beam experimentally and to observe the nonasymptotic effects in the small-angle scattering.

Small-angle scattering in the Coulomb field for the nonrelativistic case was also considered in a separate series of papers [9-12]. Regularization of the scattering amplitude was 
obtained therein by means of the Born series reconstruction, taking into account the distortion of the incident plane wave due to the long-range potential. Unlike of this approach, we consider the problem in a nonperturbative way and in the framework of the nonstationary theory in order to analyze distribution of the scattered flux.

This paper is organized as follows. In Sec. II, the main characteristic parameters of the Coulomb scattering are discussed in order to show that the asymptotic theory is not applicable in the range of small angles. In Sec. III, it is shown that the conventional asymptotic Born series for the wave function does not exist in the case of the Coulomb potential if the successive terms of the series are calculated strictly. In Sec. IV, the differential cross section is found in a nonperturbative and nonasymptotic way and it proves to be finite in the whole angular range. The polarization of the scattered electrons as well as the integral scattering characteristics are calculated in Sec. V.

\section{CHARACTERISTIC PARAMETERS}

Let us recall the conventional description of the scattering process for the stationary quantum state when the incident particle is described by the plane wave with momentum $\boldsymbol{p}$ (Fig. 1). By analogy with wave scattering in optics and radio physics, two zones may be considered. The first one (the "near" zone) can be defined as the domain where the fronts of the scattered and incident waves cannot be separated. In the second one (the "far" zone), the phase of the scattered spherical wave differs essentially from the phase of the incident plane wave and the standard asymptotic representation for the wave function can be used:

$$
\psi(\boldsymbol{r})=\left[e^{i p \cdot r}+\hat{f}\left(\boldsymbol{p}, \boldsymbol{p}^{\prime}\right) \frac{e^{i p \cdot r}}{r}\right] u(\boldsymbol{p}), \boldsymbol{p}^{\prime}=p \frac{\boldsymbol{r}}{r},
$$

where $\hat{f}\left(\boldsymbol{p}, \boldsymbol{p}^{\prime}\right)$ is the scattering amplitude operator, $r$ is the distance from the scattering center to the observation point, and $u(\boldsymbol{p})$ is a constant Dirac bispinor corresponding to a free

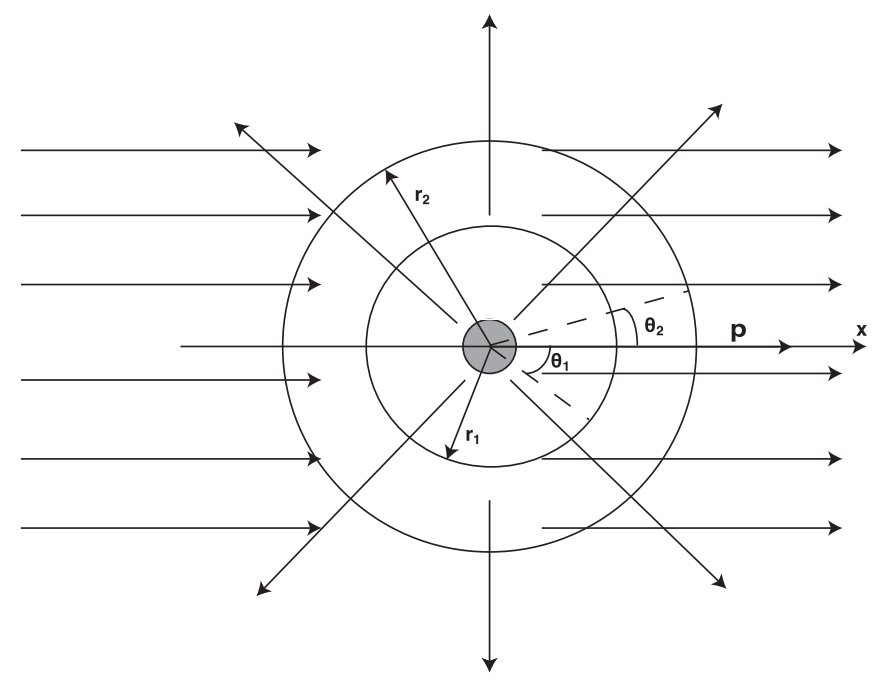

FIG. 1. Sketch of the scattering process in the stationary case. The $x$ axis is directed along the momentum $\boldsymbol{p}$ of the incident plane wave. electron. However, the asymptotic form (3) cannot be used in the near zone. The boundary $\left(\theta_{0}\right)$ between these two zones is determined from the condition that the interference between the plane and spherical waves can be neglected [6]:

$$
\begin{gathered}
p r-\boldsymbol{p} \cdot \boldsymbol{r}=2 p r \sin ^{2}(\theta / 2)>1, \\
\theta>\theta_{0} \equiv \sqrt{\frac{2}{p r}} .
\end{gathered}
$$

One can see that the angular range of the near zone depends on $r$ and is nonzero for any finite distance from the scattering center. This result is general and does not depend on the type of the potential. However, the influence of this zone on the scattering characteristics is qualitatively different for longand short-range potentials.

Let us estimate the flux $N_{\text {int }}$ of particles scattered in the domain of the near zone for both cases. For a short-range potential, it leads to

$N_{\text {int }}=2 \pi j_{0} \int_{0}^{\theta_{0}} \frac{d \sigma(\theta)}{d \Omega} \sin \theta d \theta \simeq \pi j_{0} \sigma(0) \theta_{0}^{2} \simeq 2 \pi \frac{j_{0} \sigma(0)}{p r}$,

where $j_{0}$ is the initial flux of particles. In this case the value $\sigma(0)$ is finite and the number of particles scattered in the angular range where the interference effects could be essential decreases quickly as the distance $r$ increases. Hence, their contribution to the total scattered flux can be neglected.

However, the estimation changes substantially for a longrange potential. For the Coulomb field, one can estimate the cross section at the boundary of the near zone by means of Eq. (2). That leads to

$$
\begin{gathered}
N_{\mathrm{int}}^{C}=2 \pi j_{0} \int_{\theta_{\min }}^{\theta_{0}} \frac{d \sigma(\theta)}{d \Omega} \sin \theta d \theta \simeq 4 \pi j_{0}\left(\frac{Z \alpha}{p v}\right)^{2} \frac{1}{\theta_{\min }^{2}}, \\
\theta_{0}>\theta_{\min } .
\end{gathered}
$$

The minimal scattering angle $\theta_{\min }$ can be estimated through the width of the incident wave packet:

$$
\theta_{\min } \simeq \frac{a}{r}, \quad a \simeq \frac{1}{\Delta p}
$$

where $a$ is the transversal size and $\Delta p$ is the momentum dispersion of the particle beam.

Then the number of particles scattered per unit of time within the near zone increases at long distance $r$ for the Coulomb potential,

$$
N_{\mathrm{int}}^{C} \simeq 2 \pi j_{0}\left(\frac{Z \alpha}{v}\right)^{2} r^{2}\left(\frac{1}{p a}\right)^{2} \simeq 2 \pi j_{0}\left(\frac{Z \alpha}{v}\right)^{2} r^{2}\left(\frac{\Delta p}{p}\right)^{2}
$$

and can be comparable with the number of incident particles $\left(2 \pi a^{2} j_{0}\right)$ if $p a^{2} / r<1$. This means that the contribution of these particles should be taken into account when the scattering results are analyzed. Actually, this inequality coincides with the condition that the interference effects in the scattering process could be observable within the domain where the scattered and incident particles can be still separated. It means 
that the angular boundary of the near zone should be out of the angle zone defined by the incident beam size:

$$
\theta_{\min }<\theta_{0}, \quad \frac{2 p a^{2}}{r}<1 .
$$

We recall also that the distance $r$ cannot be too large as the condition of "nonspreading" of the wave packet should be fulfilled for particle collisions [13]. In the relativistic case, this means

$$
\frac{p a^{2}}{r} \frac{p^{2}}{m^{2}}>1
$$

which can be consistent with Eq. (8).

A specific feature of relativistic particle scattering is the dependence of the polarization characteristics on the scattering angle and the asymmetry of scattering for polarized beams. If the asymptotic wave function is used, the asymmetry of the Coulomb scattering appears only in the second order of the Born series. However, it is shown below that the polarization effects in the near zone prove to be more complicated and allow one to distinguish the scattered particles.

\section{NONEXISTENCE OF THE CONVENTIONAL BORN SERIES FOR THE SOLUTION OF THE DIRAC EQUATION IN THE COULOMB FIELD}

It is a commonly used statement in textbooks that the first order of the Born series in the case of the Coulomb field leads to a modulus of the scattering amplitude that coincides with the exact result. However, let us show that this statement contradicts the result of a strict calculation of the particle wave function in the framework of perturbation theory.

The conventional Born series is the result of the recurrent solution of the integral equation that is obtained from the Dirac equation for the continuous spectrum states [2]:

$$
\psi(\boldsymbol{r})=u(\boldsymbol{p}, \varepsilon) e^{i p \cdot r}+\int g\left(\boldsymbol{r}-\boldsymbol{r}^{\prime}\right) \gamma_{4} \frac{Z \alpha}{r^{\prime}} \psi\left(\boldsymbol{r}^{\prime}\right) d \boldsymbol{r}^{\prime},
$$

where $\gamma_{v}, v=1,2,3,4$, are the Dirac matrices; $\psi(\boldsymbol{r})$ is the unknown bispinor wave function; $u(\boldsymbol{p}, \varepsilon)$ is the bispinor, corresponding to the free electron and normalized as $\bar{u} u=2 m$; and $g\left(\boldsymbol{r}-\boldsymbol{r}^{\prime}\right)$ is the Green function of the Dirac equation,

$$
g\left(\boldsymbol{r}-\boldsymbol{r}^{\prime}\right)=-\frac{1}{4 \pi}\left(\boldsymbol{\gamma} \cdot \nabla-\gamma_{4} \epsilon-m\right) \frac{e^{i p\left|r-r^{\prime}\right|}}{\left|r-r^{\prime}\right|} .
$$

The integral equation (10) is usually solved by iteration starting from $\psi^{(0)}(\boldsymbol{r})=u(\boldsymbol{p}, \epsilon) e^{i p \cdot r}$. Then the first term of this expansion (Born series) is defined as follows:

$$
\psi^{(1)}=-\frac{1}{4 \pi}\left(\boldsymbol{\gamma} \cdot \nabla-\gamma_{4} \varepsilon-m\right) \gamma_{4} u \int \frac{e^{i p\left|r-r^{\prime}\right|}}{\left|\boldsymbol{r}-\boldsymbol{r}^{\prime}\right|} \frac{Z \alpha}{r^{\prime}} e^{i p \cdot r} d \boldsymbol{r}^{\prime} .
$$

It is usually supposed that the coordinate $r$ of the observation point satisfies the inequality

$$
r \gg r^{\prime}
$$

and the following expansion is used:

$$
\left|\boldsymbol{r}-\boldsymbol{r}^{\prime}\right| \approx r-\boldsymbol{n} \cdot \boldsymbol{r}^{\prime}, \quad \boldsymbol{n}=\frac{\boldsymbol{r}}{r} .
$$

This leads to the asymptotic form for the first-order correction to the wave function:

$\psi^{(1)}=-\frac{1}{4 \pi}\left(\boldsymbol{\gamma} \cdot \nabla-\gamma_{4} \varepsilon-m\right) \gamma_{4} u \frac{e^{i p r}}{r} \int e^{i\left(p-p^{\prime}\right) \cdot r^{\prime}} \frac{Z \alpha}{r^{\prime}} d \boldsymbol{r}^{\prime}$.

However, this asymptotic expansion is correct only for a short-range potential that decreases faster than $1 / r^{s}, s \geqslant 3$ [7]. For the Coulomb case, the integration domain $V^{\prime}$ is unlimited and condition (13) is not fulfilled. Therefore, the validity of the asymptotic form (15) for the wave function should be verified.

Fortunately, in the case of the Coulomb potential the firstorder Born wave function can be calculated without asymptotic expansion of the Green function. The problem reduces to the analytical calculation of the following integral:

$$
I(\boldsymbol{r})=\frac{1}{4 \pi} \int \frac{e^{i p\left|r-r^{\prime}\right|}}{\left|\boldsymbol{r}-\boldsymbol{r}^{\prime}\right|} \frac{1}{r^{\prime}} e^{i p \cdot r^{\prime}} d \boldsymbol{r}^{\prime} .
$$

In the first step, let us apply the Fourier transformation to the Green function and the Coulomb potential and calculate the integral over $\boldsymbol{r}^{\prime}$ :

$$
\begin{gathered}
\frac{e^{i p\left|r-r^{\prime}\right|}}{\left|\boldsymbol{r}-\boldsymbol{r}^{\prime}\right|}=-\frac{1}{2 \pi^{2}} \int \frac{e^{i q \cdot\left(r-r^{\prime}\right)}}{\left(p^{2}-q^{2}+i 0\right)} d \boldsymbol{q}, \\
\frac{1}{r^{\prime}}=\frac{1}{2 \pi^{2}} \int \frac{e^{i q \cdot r^{\prime}}}{q^{2}} d \boldsymbol{q}, \\
I(\boldsymbol{r})=-\frac{1}{2 \pi^{2}} \int \frac{e^{i q \cdot r}}{\left(p^{2}-q^{2}+i 0\right)(\boldsymbol{p}-\boldsymbol{q})^{2}} d \boldsymbol{q},
\end{gathered}
$$

where the symbol $(+i 0)$ defines the correct Green function pole.

Now one can use the Feynman parametrization

$$
\frac{1}{\alpha \beta}=\int_{0}^{1} \frac{d u}{[\alpha u+\beta(1-u)]^{2}} .
$$

This leads to the following result:

$$
\begin{aligned}
I(\boldsymbol{r}) & =\frac{1}{2 \pi^{2}} \int_{0}^{1} d u \int \frac{e^{i q \cdot r}}{\left[\left(q^{2}-p^{2}-i 0\right) u+(\boldsymbol{p}-\boldsymbol{q})^{2}(1-u)\right]^{2}} d \boldsymbol{q} \\
& =\frac{1}{2 \pi^{2}} \int_{0}^{1} d u \int \frac{e^{i q \cdot r}}{\left\{[\boldsymbol{q}-\boldsymbol{p}(1-u)]^{2}-p^{2} u^{2}-i 0\right\}^{2}} d \boldsymbol{q} \\
& =\frac{1}{2 \pi^{2}} \int_{0}^{1} d u e^{i(1-u) p \cdot r} \int \frac{e^{i Q \cdot r}}{\left(Q^{2}-p^{2} u^{2}-i 0\right)^{2}} d \boldsymbol{Q} .
\end{aligned}
$$

The integral is calculated in the usual way. Introducing $\alpha$ as an angle between vectors $\boldsymbol{Q}$ and $\boldsymbol{r}$, one obtains

$$
\begin{aligned}
\int & \frac{e^{i Q \cdot r}}{\left(Q^{2}-p^{2} u^{2}-i 0\right)^{2}} d \boldsymbol{Q} \\
& =2 \pi \int_{0}^{\infty} Q^{2} d Q \int_{0}^{\pi} \frac{e^{i Q r \cos \alpha}}{\left(Q^{2}-p^{2} u^{2}\right)^{2}} \sin \alpha d \alpha \\
& =\frac{2 \pi}{i r} \int_{-\infty}^{\infty} \frac{e^{i Q r} Q}{\left(Q^{2}-p^{2} u^{2}-i 0\right)^{2}} d Q .
\end{aligned}
$$


Applying the residue theorem to (19) gives

$$
I(\boldsymbol{r})=\frac{i e^{i p \cdot r}}{2 p} \int_{0}^{1} d u \frac{e^{i(p r-p \cdot r) u}}{u} .
$$

One can see that the integral diverges logarithmically. Therefore, the conventional Born series for the wave function does not exist for any scattering angle in the case of the Coulomb field. It means that, if one uses the conventional asymptotic form (15) in order to obtain the Rutherford or Mott formula, the infinite contribution to the wave function is omitted. It was shown in Refs. [9-12] for the nonrelativistic case that this contribution is compensated if the distortion of the plane wave is taken into account. In the result, the reconstructed perturbation series was built with every term finite for all angles. Apparently, the analogous method could be also applied for the relativistic electrons. However, we consider this problem below by means of another approach based on the nonasymptotic and nonperturbative solution of the Dirac equation.

\section{DIFFERENTIAL CROSS SECTION IN THE FSM APPROXIMATION}

It is well known that the exact solution of the Dirac equation in spherical coordinates does not match the geometry of the scattering problem and the partial wave series cannot be summarized in the general case. However, there is the analytical FSM approximation [8] available in the small scattering angular range that is most interesting for us:

$$
\begin{aligned}
\Psi^{ \pm}(\mathbf{r})= & C^{ \pm} e^{i \mathbf{p r}}\left(1-\frac{i \boldsymbol{\alpha} \nabla}{2 \varepsilon}\right) \\
& \times F[ \pm i \xi, 1, i \operatorname{pr}(1-\cos \theta)] u(p),
\end{aligned}
$$

where $+(-)$ corresponds to the converging (diverging) wave, $C^{ \pm}=\Gamma(1 \mp i \xi) e^{\frac{\pi \xi}{2}}, \xi=\frac{Z \alpha \varepsilon}{p}$, and the constant bispinor amplitude $u$ is

$$
\left(\begin{array}{c}
\sqrt{\varepsilon+m} v \\
\sqrt{\varepsilon-m}(\sigma \cdot v) v
\end{array}\right)
$$

with the normalized spinor amplitude $v^{+} v=1 ; \boldsymbol{v}=\frac{p}{p}$ and $F(a, b, z)$ is the confluent hypergeometric function, $\boldsymbol{\alpha}$ are the Dirac matrices

$$
\alpha=\left(\begin{array}{ll}
0 & \sigma \\
\sigma & 0
\end{array}\right)
$$

and $\sigma$ are the Pauli matrices.

We use function (21) in order to calculate the differential cross section and the polarization characteristics of the electron scattered by a bare nucleus and to analyze the nonasymptotic effects in such collisions.

The wave function (21) describes both the scattered flux and the particles that did not change state (transition flux). In the framework of stationary scattering theory, these contributions are separated if the asymptotic form (3) of this function is used and the scattered flux is calculated with a spherical wave. However, the physical background for this separation is defined by time collision theory, as was shown in detail in Ref. [13]. Let us recall briefly this approach for conventional scattering theory because it is generalized below for the considered nonasymptotic analysis.

In real scattering processes the particle is represented as the wave packet localized near the point $\boldsymbol{r}_{0}$ at the moment $t=0$ :

$$
\begin{gathered}
\Psi(\boldsymbol{r}, 0)=\int e^{i p \cdot\left(r-r_{0}\right)} G(\boldsymbol{p}) d \boldsymbol{p}, \\
G(\boldsymbol{p})=\frac{1}{(2 \pi)^{3}} \int d \boldsymbol{r} \tilde{G}(\boldsymbol{r}) e^{-i\left(p-p_{0}\right) \cdot r}, \int d \boldsymbol{r}|\tilde{G}(\boldsymbol{r})|^{2}=1,
\end{gathered}
$$

where $G(\boldsymbol{p})$ and $\tilde{G}(\boldsymbol{r})$ define the wave-packet form in the momentum and coordinate representations, respectively. As an example, in the case of the normalized Gaussian wave packet, they have the following form:

$$
\begin{aligned}
\tilde{G}(\boldsymbol{r}) & =\left(\frac{1}{\pi a^{2}}\right)^{3 / 4} e^{-\frac{\left(r-r_{0}\right)^{2}}{2 a^{2}}}, \\
G(\boldsymbol{p}) & =\left(\frac{a^{2}}{\pi}\right)^{3 / 4} e^{-\frac{\left(p-p_{0}\right)^{2}}{2} a^{2}},
\end{aligned}
$$

with $a$ as the space width of the packet and $\boldsymbol{p}_{0}$ as its central momentum.

If one uses the asymptotic expression (3) for the stationary solution of the Dirac equation, the wave-packet evolution is described as follows:

$$
\Psi(\boldsymbol{r}, t)=\int\left[e^{i p \cdot r}+\hat{f}\left(\boldsymbol{p}, \boldsymbol{p}^{\prime}\right) \frac{e^{i p r}}{r}\right] u(\boldsymbol{p}) G(\boldsymbol{p}) e^{-i \varepsilon(p) t-i p \cdot r_{0}} d \boldsymbol{p} .
$$

One of the main results of the asymptotic theory is that the observable scattering characteristics are defined by the potential properties only and do not depend on the form of the packet [13]. Mathematically, this follows from the assumption that the function $\hat{f}\left(\boldsymbol{p}, \boldsymbol{p}^{\prime}\right)$ is rather smooth in comparison with the function $G(\boldsymbol{p})$. Then one can use the following approximation:

$$
\begin{gathered}
\boldsymbol{p}=\boldsymbol{p}_{0}+\boldsymbol{q}, \quad|\boldsymbol{q}| \ll\left|\boldsymbol{p}_{0}\right|, \\
p=\left|\boldsymbol{p}_{0}+\boldsymbol{q}\right| \approx p_{0}+\frac{\boldsymbol{p}_{0}}{p_{0}} \cdot \boldsymbol{q}, \\
p^{2} \approx p_{0}^{2}+2 \boldsymbol{p}_{0} \cdot \boldsymbol{q},
\end{gathered}
$$

and one can represent the wave packet (25) as

$$
\begin{aligned}
& \Psi(\boldsymbol{r}, t)= {\left[e^{i p_{0} \cdot r} \tilde{G}\left(\boldsymbol{r}-\boldsymbol{v}_{0} t-\boldsymbol{r}_{0}\right)+\hat{f}\left(\boldsymbol{p}_{0}, \boldsymbol{p}_{0}^{\prime}\right) \frac{e^{i p_{0} r}}{r} \tilde{G}\right.} \\
&\left.\times\left(r \frac{\boldsymbol{p}_{0}}{p_{0}}-\boldsymbol{v}_{0} t-\boldsymbol{r}_{0}\right)\right] u\left(\boldsymbol{p}_{0}\right) e^{-i \varepsilon\left(p_{0}\right) t}, \\
&\left.\frac{\partial \varepsilon}{\partial \boldsymbol{p}}\right|_{p=p_{0}}=\boldsymbol{v}_{0} .
\end{aligned}
$$

Here the first term describes the spreading of the "incident" wave and the second one corresponds to the "scattered" wave. They are clearly separated in space if a localized wave packet is considered. As an example, if a coordinate system with the $x$ axis directed along $\boldsymbol{v}_{0}$ and the Gaussian 


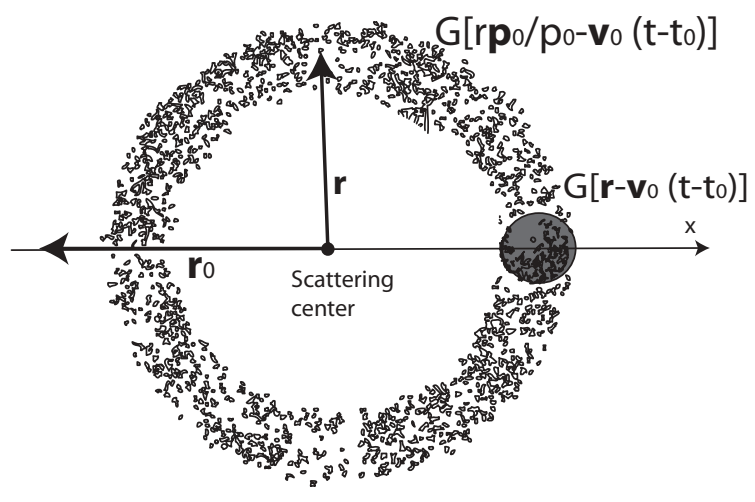

FIG. 2. The probability distribution for the scattered and incident wave packets.

wave packet are used, the function $\tilde{G}$ takes the following form:

$$
\begin{gathered}
\tilde{G}\left(\boldsymbol{r}-\boldsymbol{v}_{0}\left(t-t_{0}\right)\right) \sim e^{-\frac{\left|r-v_{0}\left(t-t_{0}\right)\right|^{2}}{a^{2}}}=e^{-\frac{\left[x-v_{0}\left(t-t_{0}\right)\right]^{2}}{2 a^{2}}} e^{-\frac{r_{\perp}^{2}}{2 a^{2}},} \\
\tilde{G}\left(r \frac{\boldsymbol{p}_{0}}{p_{0}}-\boldsymbol{v}_{0}\left(t-t_{0}\right)\right) \sim e^{-\frac{\left[r-v_{0}\left(t-t_{0}\right)\right]^{2}}{2 a^{2}}}, \\
\boldsymbol{r}_{0}=-x_{0} \frac{\boldsymbol{v}_{0}}{v_{0}}, t_{0}=\frac{x_{0}}{v_{0}},
\end{gathered}
$$

where $r_{\perp}$ is the transverse distance from the $x$ axis. This means that the incident part of the packet is negligible if $r_{\perp} \approx r \theta>a$, that is, $\theta>a / r$. At the same time the scattered wave packet is localized in the whole spherical shell with radius $r$ and width $a$. Figure 2 shows the sketch of the packet propagation. In the dotted region, the flux is defined only by the scattered wave packet. In this case the differential cross section is equal to $\left|\hat{f}\left(\boldsymbol{p}_{0}, \boldsymbol{p}_{0}^{\prime}\right)\right|^{2}$ independently of the wave-packet form and with the scattering amplitude calculated from the stationary scattering theory.

However, these arguments cannot be applied directly to the Coulomb potential because the scattering amplitude has a nonintegrable singularity in this case and formula (27) is incorrect. For the nonrelativistic case, one can analyze the wave-packet evolution if Eq. (25) is generalized as follows (see Ref. [6]):

$$
\begin{aligned}
\Psi(\boldsymbol{r}, t)= & C \int e^{i p \cdot r} F[i \xi, 1, i(p r-\boldsymbol{p} \cdot \boldsymbol{r})] \\
& \times G(\boldsymbol{p}) e^{-i \varepsilon(p) t-i p \cdot r_{0}} d \boldsymbol{p} .
\end{aligned}
$$

Here $F(a, b, i z)$ is the confluent hypergeometric function that defines the exact solution of the Schrödinger equation, and $C=e^{\pi \xi / 2} \Gamma(1-i \xi)$ is the normalization constant; $z=p r-$ $\boldsymbol{p} \cdot \boldsymbol{r}$.

The function $F(a, b, i z)$ can be represented as a sum of two hypergeometric functions of the third genus, $U_{1,2}(a, b, i z)[14]$ :

$$
F(a, b, i z)=\frac{\Gamma(b)}{\Gamma(b-a)} U_{2}(a, b, i z)+\frac{\Gamma(b)}{\Gamma(a)} U_{1}(a, b, i z) .
$$

This well-known expansion is usually used for the asymptotic analysis of Coulomb scattering [1] because in the far zone $(z \gg 1), U_{2}( \pm i \xi, 1, i z)$ leads to the plane-wave function and $U_{1}( \pm i \xi, 1, i z)$ leads to the spherical wave function with the coefficient that defines the scattering amplitude. As in Ref. [14], both functions of the third genus in the expansion (30) have logarithmic singularities in the near zone $(z<1)$. However, as was shown in Ref. [6], the expansion (30) can be used in Eq. (29) in the whole angular range because these singularities are integrable. There it was proved that the scattered flux could be calculated using only the function $U_{1}(i \xi, 1, i z)$ and remained finite and independent of the form of the wave packet within the whole range of scattering angles if condition (9) was fulfilled.

One can try to use the same approach for relativistic Coulomb scattering. In the FSM approximation, the wave function (21) can be represented as the sum of two functions:

$$
\begin{gathered}
\Psi_{p}^{+}(\boldsymbol{r})=\Psi^{(1)}(\boldsymbol{r})+\Psi^{(2)}(\boldsymbol{r}), \\
\Psi^{(1)}(\boldsymbol{r})=C e^{i p \cdot r} F(i \xi, 1, i z) u(\boldsymbol{p}), \xi=\frac{Z \alpha \varepsilon}{p},
\end{gathered}
$$

$$
\begin{aligned}
\Psi^{(2)}(\boldsymbol{r}) & =-i C e^{i p \cdot r} \frac{\boldsymbol{\alpha} \cdot \nabla}{2 \varepsilon} F(i \xi, 1, i z) u(\boldsymbol{p}) \\
& =i \frac{\xi}{2 \varepsilon} C e^{i p \cdot r} \frac{\boldsymbol{\alpha} \cdot(p \boldsymbol{r}-r \boldsymbol{p})}{r} F(i \xi+1,2, i z) u(\boldsymbol{p}) .
\end{aligned}
$$

The function $\Psi^{(1)}$ has the same form as for the nonrelativistic case, and only the part of it corresponding to $F(i \xi, 1, i z) \rightarrow \frac{1}{\Gamma(i \xi)} U_{1}(i \xi, 1, i z)$ can be used for calculation of the scattering flux analogously to Ref. [6].

However, this approach proves to be inapplicable to the function $\Psi^{(2)}(\boldsymbol{r})$ that is specific to the relativistic case. If one uses expansion (30) for $F(i \xi+1,2, i z)$, the function $U_{1}(i \xi+$ $1,2, i z$ ) has a nonintegrable singularity $\sim 1 / z$ as $z \rightarrow 0$ and it is not possible to separate the scattered flux throughout the whole angular range. Therefore, an additional analysis of the wave-packet evolution is required for the relativistic problem. Let us apply the stationary states (21) at the wave packet (29),

$$
\Psi(\boldsymbol{r}, t)=\int \Psi_{p}^{+}(\boldsymbol{r}) G(\boldsymbol{p}) e^{-i \varepsilon(p) t-i p \cdot r_{0}} d \boldsymbol{p} .
$$

It is evident that the bispinor $u(\boldsymbol{p})$ is a rather smooth function of the momentum and it can be referred to the central momentum $\boldsymbol{p}_{0}$ of the wave packet. This allows us to take into account the polarization characteristics of the process before the separation of the scattered and incident waves.

Let us represent the wave function (31) in the following form:

$$
\begin{gathered}
\Psi(\boldsymbol{r}, t)=\left[\Phi(\boldsymbol{r}, t)+i p_{0} \Phi_{1}(\boldsymbol{r}, t) \boldsymbol{\alpha} \cdot\left(\boldsymbol{n}-\boldsymbol{v}_{0}\right)\right] u\left(\boldsymbol{p}_{0}\right), \\
\boldsymbol{n}=\frac{\boldsymbol{r}}{r}, \quad \boldsymbol{v}_{0}=\frac{\boldsymbol{p}_{0}}{p_{0}}, \\
\Phi(\boldsymbol{r}, t)=C \int F(i \xi, 1, i z) G(\boldsymbol{p}, t) d \boldsymbol{p}, \\
\Phi_{1}(\boldsymbol{r}, t)=C \frac{\xi}{2 \varepsilon\left(p_{0}\right)} \int F(i \xi+1,2, i z) G(\boldsymbol{p}, t) d \boldsymbol{p}, \\
G(\boldsymbol{p}, t)=G(\boldsymbol{p}) e^{-i \varepsilon(p) t+i p \cdot\left(r-r_{0}\right)},
\end{gathered}
$$

with the functions $\Phi(\boldsymbol{r}, t)$ and $\Phi_{1}(\boldsymbol{r}, t)$, which do not depend on the spin variables. 
We use definition (22) in order to represent wave function (32) in a form that can be compared with the conventional description of the relativistic scattering [2],

$$
\Psi=\left(\begin{array}{c}
\sqrt{\varepsilon_{0}+m} T_{1} v \\
\sqrt{\varepsilon_{0}-m}(\boldsymbol{\sigma} \cdot \boldsymbol{n}) T_{2} v
\end{array}\right),
$$

where $T_{1}$ and $T_{2}$ are the scattering operators

$$
\begin{aligned}
T_{1}=\Phi & +i\left(\varepsilon_{0}-m\right) \Phi_{1}\left[(\boldsymbol{\sigma} \cdot \boldsymbol{n})\left(\boldsymbol{\sigma} \cdot \boldsymbol{v}_{0}\right)-1\right] \\
T_{2}= & \Phi(\boldsymbol{\sigma} \cdot \boldsymbol{n})\left(\boldsymbol{\sigma} \cdot \boldsymbol{v}_{0}\right) \\
& +i\left(\varepsilon_{0}+m\right) \Phi_{1}\left[1-(\boldsymbol{\sigma} \cdot \boldsymbol{n})\left(\boldsymbol{\sigma} \cdot \boldsymbol{v}_{0}\right)\right]
\end{aligned}
$$

Let us suppose that the polarization of the electron in the initial state is described by the density matrix operator

$$
\rho_{\alpha \beta} \equiv v_{\alpha} v_{\beta}^{*}=\frac{1}{2}(1+\zeta \cdot \sigma)_{\alpha \beta},
$$

where $\zeta$ is the electron polarization vector (average spin of the electron in the reference frame where it is in rest).

The wave packet (32) allows one to calculate the probability for the particle with arbitrary polarization to cross the area of the detector $d \Sigma=r^{2} d \Omega$ in a unit of time and the polarization vector $\zeta^{\prime}$ for the electron in the final state:

$$
\begin{gathered}
d I=(\boldsymbol{j} \cdot \boldsymbol{n}) r^{2} d \Omega, \\
\boldsymbol{\zeta}^{\prime}=\operatorname{Tr}\left(\rho^{\prime} \boldsymbol{\sigma}\right),
\end{gathered}
$$

where $\boldsymbol{j}$ includes both the incident and scattered parts of the wave packet and the prime indicates a final state. Using the definition of the Dirac flux, $j=\Psi^{*} \alpha \Psi$, and taking into account the identity $(\boldsymbol{\sigma} \cdot \boldsymbol{A})(\boldsymbol{\sigma} \cdot \boldsymbol{B})=(\boldsymbol{A} \cdot \boldsymbol{B})+i \boldsymbol{\sigma} \cdot[\boldsymbol{A} \times \boldsymbol{B}]$, one can obtain

$$
\begin{aligned}
d I= & 2 p_{0} \operatorname{Sp}\left(T_{1} \rho T_{2}^{\dagger}+T_{2} \rho T_{1}^{\dagger}\right) r^{2} d \Omega=\left\{|\Phi|^{2} \boldsymbol{n} \cdot \boldsymbol{v}_{0}\right. \\
& +\left[2 \varepsilon_{0} \operatorname{Im}\left(\Phi \Phi_{1}^{*}\right)-2 p_{0}^{2}\left|\Phi_{1}\right|^{2}\right]\left(1-\boldsymbol{n} \cdot \boldsymbol{v}_{0}\right) \\
& \left.+2 m \operatorname{Re}\left(\Phi \Phi_{1}^{*}\right) \zeta \cdot \boldsymbol{\tau}\right\} r^{2} d \Omega \\
& \boldsymbol{\tau}=\boldsymbol{n} \times \boldsymbol{v}_{0}, \quad \varepsilon_{0}=\varepsilon\left(\boldsymbol{p}_{0}\right) .
\end{aligned}
$$

The polarization of the final electron state is different from that of the initial state and is described by the density matrix defined as follows [2]:

$$
\rho^{\prime}=\frac{T_{1} \rho T_{2}^{\dagger}+T_{2} \rho T_{1}^{\dagger}}{\operatorname{Tr}\left(T_{1} \rho T_{2}^{\dagger}+T_{2} \rho T_{1}^{\dagger}\right)} .
$$

Substituting Eq. (38) into Eq. (36) and using expression (34) for the scattering operators, one can obtain

$$
\boldsymbol{\zeta}^{\prime}=\frac{g \boldsymbol{\tau}+f \zeta+c \zeta \times \boldsymbol{\tau}+e \boldsymbol{n}\left(\boldsymbol{\tau}\left[\zeta \times \boldsymbol{v}_{0}\right]\right)-e \boldsymbol{n} \times \boldsymbol{\tau}\left(\boldsymbol{\zeta} \cdot \boldsymbol{v}_{0}\right)}{f+g \zeta \cdot \boldsymbol{\tau}},
$$

where

$$
\begin{gathered}
g=2 m \operatorname{Re}\left(\Phi \Phi_{1}^{*}\right), \\
f=|\Phi|^{2} \boldsymbol{n} \cdot \boldsymbol{v}_{0}+2 \varepsilon_{0} \operatorname{Im}\left(\Phi \Phi_{1}^{*}\right)\left(1-\boldsymbol{n}_{0} \cdot \boldsymbol{v}_{0}\right) \\
-2 p_{0}^{2}\left|\Phi_{1}\right|^{2}\left(1-\boldsymbol{n} \cdot \boldsymbol{v}_{0}\right), \\
c=|\Phi|^{2}-2 \varepsilon_{0} \operatorname{Im}\left(\Phi \Phi_{1}^{*}\right)-2 p_{0}^{2}\left|\Phi_{1}\right|^{2}, \\
e=2 p_{0}^{2}\left|\Phi_{1}\right|^{2}-2\left(\varepsilon_{0}-m\right) \operatorname{Im}\left(\Phi \Phi_{1}^{*}\right) .
\end{gathered}
$$

The polarization is a complicated function of $r$ as well as a differential cross section. If the initial electron is not polarized (i.e., $\zeta=0$ ), the scattering electron still will obtain a certain polarization. This contradicts the Mott formula (2). (In the conventional asymptotic theory, polarization appears only in the second-order term of the Born series.) The number of polarized particles which appear as the result of scattering of a nonpolarized beam is usually described with the asymmetry of scattering, $\Delta(\theta)$ :

$$
\zeta^{\prime}=\Delta(\theta) \tau, \quad \Delta(\theta)=\frac{g}{f}
$$

In order to find the scattering cross section, one should pass to the stationary flux of the incident particles instead of the one-particle wave packet. According to Ref. [13], in this case the initial coordinates $\boldsymbol{r}_{0}$ should be considered as uniformly distributed with the density $\rho_{0}$ in space and the flux is integrated over these coordinates. As an example, one can find for the incident flux

$$
\begin{aligned}
\boldsymbol{j}_{0} & =\rho_{0} \int \Psi_{0}^{*}(\boldsymbol{r}) \boldsymbol{\alpha} \Psi_{0}(\boldsymbol{r}) d \boldsymbol{r}_{0} \\
& =\rho_{0} \int\left|G\left(\boldsymbol{r}-\boldsymbol{r}_{0}\right)\right|^{2} \bar{u}\left(\boldsymbol{p}_{0}\right) \boldsymbol{\alpha} u\left(\boldsymbol{p}_{0}\right) d \boldsymbol{r}_{0}=2 \boldsymbol{p}_{0} \rho_{0},
\end{aligned}
$$

where $\Psi_{0}$ is a solution of the free Dirac equation.

As one can see, the scattering flux in Eq. (37) includes four terms:

$$
\begin{gathered}
j_{1}=|\Phi|^{2} \boldsymbol{n} \cdot \boldsymbol{v}_{0}, \quad j_{2}=2 \varepsilon_{0} \operatorname{Im}\left(\Phi \Phi_{1}^{*}\right)\left(1-\boldsymbol{n} \cdot \boldsymbol{v}_{0}\right), \\
j_{3}=2 m \operatorname{Re}\left(\Phi \Phi_{1}^{*}\right)(\zeta \cdot \boldsymbol{\tau}), \quad j_{4}=2 p_{0}^{2}\left|\Phi_{1}\right|^{2}\left(1-\boldsymbol{n} \cdot \boldsymbol{v}_{0}\right) .
\end{gathered}
$$

Each of these terms is finite at any scattering angle because the functions $\Phi$ and $\Phi_{1}$ in Eq. (32) are well defined in the entire range of their arguments. The problem is how to separate the scattered flux and to exclude the dependence on the wave-packet form in the values in Eqs. (42) when calculating the scattering cross section. As was mentioned above, this problem was solved in Ref. [6] for nonrelativistic particles using the expansion (30) for the hypergeometric function. According to the definition in Eq. (32), the value $j_{1}$ is defined by the same hypergeometric function as in the nonrelativistic case. This means that, in the first term of Eq. (37), the scattered flux is calculated by substitution of the hypergeometric functions of the second kind, $1 / \Gamma(i \xi) U_{1}(i \xi, 1, i z)$, instead of $F(i \xi, 1, i z)$ :

$$
\begin{aligned}
\frac{d I^{(1)}}{r^{2} d \Omega} & =\frac{2\left(\boldsymbol{n} \cdot \boldsymbol{p}_{0}\right)|C|^{2}}{|\Gamma(i \xi)|^{2}}\left|\int F(i \xi, 1, i z) G(\boldsymbol{p}, t) d \boldsymbol{p}\right|^{2} \\
\rightarrow \frac{d I_{\mathrm{sc}}^{(1)}}{r^{2} d \Omega} & =\frac{2\left(\boldsymbol{n} \cdot \boldsymbol{p}_{0}\right)|C|^{2}}{|\Gamma(i \xi)|^{2}}\left|\int U_{1}(i \xi, 1, i z) G(\boldsymbol{p}, t) d \boldsymbol{p}\right|^{2} .
\end{aligned}
$$

As was shown in Ref. [6], if one uses the integral representation [14] for the function $U_{1}(i \xi, 1, i z)$ and Eq. (32) 
for $G(\boldsymbol{p}, t)$, the integral in Eq. (43) transforms as follows:

$$
\begin{gathered}
\int U_{1}(i \xi, 1, i z) G(\boldsymbol{p}, t) d \boldsymbol{p} \\
\simeq U_{1}\left(i \xi, 1, i z_{0}\right) e^{i p_{0} \cdot\left(r-r_{0}\right)} \tilde{G}\left(r \frac{\boldsymbol{p}_{0}}{p_{0}}-\boldsymbol{v}_{0} t-\boldsymbol{r}_{0}\right) e^{-i \varepsilon\left(p_{0}\right) t}, \\
z_{0}=p_{0} r-\boldsymbol{p}_{0} \cdot \boldsymbol{r} .
\end{gathered}
$$

This corresponds to the spherical wave packet in Fig. 2 with the amplitude defined by the function $U_{1}\left(i \xi, 1, i z_{0}\right)$ referring to the central momentum of the packet. When stationary conditions are considered, the scattered flux should be integrated over $\boldsymbol{r}_{0}$ as in Eq. (41) and the following contribution to the cross section can be found independently of the form of the wave packet:

$$
\begin{aligned}
\frac{d \sigma^{(1)}}{d \Omega}= & \frac{r^{2}}{j_{0}} \rho_{0} \int d \boldsymbol{r}_{0} \frac{d I_{\mathrm{sc}}^{(1)}}{d \Omega} \\
= & \frac{r^{2}}{j_{0}} \rho_{0}\left(\boldsymbol{n} \cdot \boldsymbol{p}_{0}\right) \frac{|C|^{2}}{|\Gamma(i \xi)|^{2}}\left|U_{1}\left(i \xi, 1, i z_{0}\right)\right|^{2} \\
& \times \int d \boldsymbol{r}_{0}\left|\tilde{G}\left(r \frac{\boldsymbol{p}_{0}}{p_{0}}-\boldsymbol{v}_{0} t-\boldsymbol{r}_{0}\right)\right|^{2} \\
= & \left(\boldsymbol{n} \cdot \boldsymbol{v}_{0}\right) \frac{|C|^{2}}{|\Gamma(i \xi)|^{2}}\left|U_{1}\left(i \xi, 1, i z_{0}\right)\right|^{2} r^{2}
\end{aligned}
$$

This value still has a logarithmic singularity since $\left|U_{1}\left(i \xi, 1, i z_{0}\right)\right|^{2} \sim \ln ^{2} z_{0} \sim \ln ^{2} \theta, \theta \rightarrow 0$ [14]. It is conditioned by the fact that the incident and scattered fluxes are not separated for angles $\theta<\theta_{\min }$ from Eq. (6), where the total flux should be calculated with the function $F(i \xi, 1, i z)$. However, the singularity in Eq. (45) is integrable and the contribution of the scattered flux over the angle range $\theta<\theta_{\min }$ is $\sim$ $\theta_{\min }^{2} \ln ^{2} \theta_{\min }<\theta_{0}^{2} \ll 1$. It is negligible when analyzing the cross section within the whole near zone, $\theta<\theta_{0}$, because of inequality (8). This means that Eq. (45) is available in the entire range of the scattering angle analogously to the nonrelativistic case [6].

In the same way, one can calculate the contributions to the cross section from the second and third terms in Eq. (42):

$$
\begin{aligned}
\frac{d \sigma^{(2)}}{d \Omega}= & \frac{r^{2}}{j_{0}} \rho_{0} \int d \boldsymbol{r}_{0} \frac{d I_{\mathrm{sc}}^{(2)}}{d \Omega}=\xi r^{2}\left(1-\boldsymbol{n} \cdot \boldsymbol{v}_{0}\right)|C|^{2} \mathrm{Im} \\
& \times\left[\frac{U_{1}(i \xi, 1, i z) U_{1}^{*}(i \xi+1,2, i z)}{\Gamma(i \xi) \Gamma^{*}(i \xi+1)}\right], \\
\frac{d \sigma^{(3)}}{d \Omega}= & \frac{r^{2}}{j_{0}} \rho_{0} \int d \boldsymbol{r}_{0} \frac{d I_{\mathrm{sc}}^{(3)}}{d \Omega}=r^{2} \xi \frac{m}{\varepsilon_{0}}\left(\zeta \cdot \boldsymbol{\tau}_{0}\right)|C|^{2} \operatorname{Re} \\
& \times\left[\frac{U_{1}(i \xi, 1, i z) U_{1}^{*}(i \xi+1,2, i z)}{\Gamma(i \xi) \Gamma^{*}(i \xi+1)}\right] .
\end{aligned}
$$

In spite of the more singular behavior of the function $U_{1}(i \xi+1,2, i z) \sim 1 / z, z \rightarrow 0$, the cross sections $(46) \sim \ln z$ because of the term $\left(1-\boldsymbol{n} \cdot \boldsymbol{v}_{0}\right) \sim z$. The value in Eq. (47) $\sim \zeta \ln z / \sqrt{z}$. It means that both contributions remain integrable at small angles. Thus, they are also available in the entire range of the scattering angle and do not depend on the wave-packet form in the case of stationary incident flux.
However, the cross section conditioned by the scattered flux $j_{4}$ in Eq. (42) cannot be considered this way because (1 $\left.\boldsymbol{n} \cdot \boldsymbol{v}_{0}\right)\left|U_{1}(i \xi+1,2, i z)\right|^{2} \sim 1 / z, z \rightarrow 0$, has a nonintegrable singularity. Hence, at small angles, Eq. (30) is not available and this term should be calculated with the hypergeometric function. It includes the following integral:

$$
I_{0}=\left|\int F[i \xi+1,2, i(p r-\boldsymbol{p} \cdot \boldsymbol{r})] G(\boldsymbol{p}, t) d \boldsymbol{p}\right|^{2} .
$$

Using the expansion (26) one can find

$$
\begin{aligned}
I_{0}= & \mid \int e^{i q \cdot\left(r-v_{0} t-r_{0}\right)} F[i \xi+1,2, i \\
& \left.\times\left(z_{0}+\frac{q_{\perp}^{2} r}{2 p_{0}}+r \frac{\boldsymbol{p}_{0}}{p_{0}} \cdot \boldsymbol{q}-\boldsymbol{q} \cdot \boldsymbol{r}\right)\right]\left.G(\boldsymbol{q}) d \boldsymbol{q}\right|^{2},
\end{aligned}
$$

where the quadratic term $q_{\perp}^{2} / 2 p_{0}$ should be taken into account in the case of small scattering angles, and $q_{\perp}$ is an absolute value of vector which is orthogonal to $\boldsymbol{q}$.

Let us choose the Gaussian form of the wave packet and the coordinate system in accordance with Eqs. (24) and (28) in order to make the further analysis more clear. In this case Eq. (49) transforms as follows:

$$
\begin{gathered}
I_{0}=N \mid \int F\left\{i \xi+1,2, i\left[z_{0}+\frac{q_{\perp}^{2} r}{2 p_{0}}+q_{x}(r-x)-\boldsymbol{q}_{\perp} \cdot \boldsymbol{r}_{\perp}\right]\right\} \\
\times\left. e^{-\frac{q_{\perp}^{2} a^{2}}{2}+i q_{\perp} \cdot r_{\perp}} e^{-\frac{q_{x}^{2} a^{2}}{2}+i q_{x}\left[x-v_{0}\left(t-t_{0}\right)\right]} d \boldsymbol{q}_{\perp} d q_{x}\right|^{2} \\
N=\frac{a^{3}}{(2 \pi)^{3}(\pi)^{3 / 2}}, \quad\left(\boldsymbol{q}_{\perp} \cdot p_{0}\right)=0 .
\end{gathered}
$$

In the entire angular range, the value $q_{x}(r-x) \ll z_{0}=$ $p_{0}(r-x)$ because of $\left|q_{x}\right| \sim 1 / a \ll p_{0}$. Hence, one can integrate over $q_{x}$ in Eq. (50), putting $q_{x}=0$ in the hypergeometric function argument

$$
\begin{aligned}
I_{0}= & N \frac{2 \pi}{a^{2}} e^{-\frac{\left[x-v_{0}\left(t-t_{0}\right)\right]^{2}}{a^{2}}} \mid \int F\left[i \xi+1,2, i\left(z_{0}+\frac{q_{\perp}^{2} r}{2 p_{0}}\right.\right. \\
& \left.\left.-\boldsymbol{q}_{\perp} \cdot \boldsymbol{r}_{\perp}\right)\right]\left.e^{i q_{\perp} \cdot r_{\perp}-\frac{q_{\perp}^{2} a^{2}}{2}} d \boldsymbol{q}_{\perp}\right|^{2} .
\end{aligned}
$$

Let us now consider the integral

$$
\begin{aligned}
A(\theta)= & \int_{0}^{\infty} q_{\perp} e^{-\frac{q_{\perp}^{2} a^{2}}{2}} d q_{\perp} \int_{0}^{2 \pi} d \varphi e^{i q_{\perp} r_{\perp} \cos \varphi} \\
& \times F\left[i\left(z_{0}+\frac{q_{\perp}^{2} r}{2 p_{0}}-q_{\perp} r_{\perp} \cos \varphi\right)\right],
\end{aligned}
$$

where $\phi$ is the angle between $\boldsymbol{r}_{\perp}$ and $\boldsymbol{q}_{\perp}$. It is most important to analyze it for small angles comparable with the width of the near zone. In this case, the dimensionless variables $\eta$ and $y$ are convenient:

$$
\begin{gathered}
\eta=\frac{\theta}{\theta_{0}}, \quad \theta_{0}=\sqrt{\frac{2}{p r}}, \\
\cos \theta \sim 1-\theta^{2} / 2, \quad \sin ^{2} \theta / 2 \sim \theta^{2} / 4,
\end{gathered}
$$




$$
\begin{gathered}
z_{0} \approx p_{0} r \frac{\theta^{2}}{2}=\eta^{2}, \quad \theta \approx \sqrt{\frac{2 z_{0}}{p_{0} r}}, \\
q_{\perp}=\frac{y}{a}, r_{\perp}=r \sin \theta \sim r \theta, \boldsymbol{q}_{\perp} \cdot \boldsymbol{r}_{\perp}=\frac{r}{a} \theta_{0} y \eta \cos \varphi .
\end{gathered}
$$

Taking into account the definition of the angle $\theta_{\min }$ with Eq. (6),

$$
\begin{gathered}
\boldsymbol{q}_{\perp} \cdot \boldsymbol{r}_{\perp} \approx \frac{r}{a} \theta_{0} y \eta \cos \varphi=\delta y \eta \cos \varphi, \\
\frac{q_{\perp}^{2} r}{2 p_{0}}=\frac{y^{2} r}{2 a^{2} p_{0}}=\frac{y^{2} \delta^{2}}{4}, \\
\delta=\frac{r}{a} \theta_{0}=\frac{\theta_{0}}{\theta_{\min }} \gg 1,
\end{gathered}
$$

the integral (52) can be transformed as follows:

$$
\begin{aligned}
A(\theta)= & \frac{1}{a^{2}} \int_{0}^{\infty} y e^{-\frac{y^{2}}{2}} d y \int_{0}^{2 \pi} e^{i \delta \eta y \cos \varphi} F[i \xi+1,2, i \\
& \left.\times\left(\eta^{2}-\delta \eta y \cos \varphi+\frac{y^{2} \delta^{2}}{4}\right)\right] d \varphi .
\end{aligned}
$$

Evidently, this value is finite because it includes only the convergent integrals, and the result of integration in Eq. (55) will depend on the width of the wave packet of which the form is not controlled in the experiment. However, the contribution of this flux to the total cross section proves to be not very sensitive to precise values of the wave-packet parameters because it depends on them only logarithmically. This allows one to take the function $F\left[i \xi+1,2, i\left(v^{2}-\delta \eta y \cos \varphi+\frac{y^{2} \delta^{2}}{4}\right)\right]$ out of the integral at some "average" values for $y$ and $\varphi$. Then the term proportional to $y$ is close to zero because of averaging over $\varphi$ uniformly distributed in the interval $0<\varphi<2 \pi$. The quadratic term can be approximated as

$$
\frac{y^{2} \delta^{2}}{4} \approx \frac{\tilde{\delta}^{2}}{2}, \quad \tilde{\delta} \approx \frac{r}{\tilde{a}} \theta_{0}=\sqrt{\frac{2 r}{p_{0} \tilde{a}^{2}}},
$$

depending on the average space width $\tilde{a}$ of the wave packet.

Then one can separate the contributions of the scattered and incident fluxes in Eq. (55) by means of the expansion (30). This results in

$$
\begin{aligned}
A_{\mathrm{sc}}= & \frac{1}{a^{2} \Gamma(1+i \xi)} U_{1}\left[i \xi+1,2, i\left(\eta^{2}+\frac{\tilde{\delta}^{2}}{2}\right)\right] \\
& \times \int_{0}^{\infty} y e^{-\frac{y^{2}}{2}} d y \int_{0}^{2 \pi} e^{i \delta \eta y \cos \varphi} d \varphi .
\end{aligned}
$$

This leads to the scattering cross section as an integrable function of angle, because the function $U_{1}\left[i \xi+1,2, i\left(\eta^{2}+\right.\right.$ $\left.\left.\frac{\tilde{\delta}^{2}}{2}\right)\right] \sim 1 / \tilde{\delta}^{2}, \eta \rightarrow 0$, instead of $U_{1}\left(i \xi+1,2, i \eta^{2}\right) \sim 1 / \eta^{2}$, $\eta \rightarrow 0$, if the wave packet is not taken into account.

Then the last contribution to the scattering cross section for the stationary incident flux can be calculated in a form analogous to Eqs. (45) and (47):

$$
\begin{aligned}
\frac{d \sigma^{(4)}}{d \Omega}= & -\frac{r^{2}}{2} \xi^{2} v_{0}^{2}\left(1-\boldsymbol{n} \cdot \boldsymbol{v}_{0}\right)|C|^{2} \mid U_{1}[i \xi+1,2, i \\
& \left.\times\left(\eta^{2}+\frac{\tilde{\delta}^{2}}{2}\right)\right]\left.\right|^{2} \frac{1}{|\Gamma(i \xi+1)|^{2}} .
\end{aligned}
$$

Let us now write the final result for the differential scattering cross section and the polarization asymmetry for an unpolarized incident electron beam $(\zeta=0)$. In this case the result does not depend on the azimuthal angle, and the dimensionless variable can be used for analysis of the small-angle range

$$
\theta=\theta_{0} \eta=\eta \sqrt{\frac{2}{p_{0} r}}, \quad \sin \theta d \theta \approx \frac{2}{p_{0} r} \eta d \eta .
$$

Then one finds

$$
\begin{aligned}
\frac{d \sigma}{d \eta}= & \frac{4 \pi r}{p_{0}} \eta|C|^{2}\left\{\left(1-\frac{\eta^{2}}{p_{0} r}\right) \frac{\left|U_{1}\left(i \xi, 1, i \eta^{2}\right)\right|^{2}}{|\Gamma(i \xi)|^{2}}\right. \\
& +\xi \frac{\eta^{2}}{p_{0} r} \operatorname{Im}\left[\frac{U_{1}\left(i \xi, 1, i \eta^{2}\right) U_{1}^{*}\left(i \xi+1,2, i \eta^{2}\right)}{\Gamma(i \xi) \Gamma^{*}(i \xi+1)}\right] \\
& \left.-\xi^{2} \frac{\eta^{2} v_{0}^{2}}{2 p_{0} r} \frac{1}{|\Gamma(i \xi+1)|^{2}} \mid U_{1}\left[i \xi+1,2, i\left(\eta^{2}+\frac{\tilde{\delta}^{2}}{2}\right)\right]^{2}\right\} \\
\equiv & \frac{4 \pi r}{p_{0}} \eta|C|^{2} \tilde{f} .
\end{aligned}
$$

This value should be compared with Mott's formula (2) written in the same variables:

$$
\frac{d \sigma}{d \eta}=\frac{4 \pi r}{p_{0}} \xi^{2}\left(1-\frac{v_{0}^{2} \eta^{2}}{2 p_{0} r}\right) \frac{1}{\eta^{3}} .
$$

It can be verified that in the far zone when $\eta \gg 1,1 \gg$ $\theta \gg \theta_{0}$, where the asymptotics of the functions $U_{1}$ can be used, both formulas coincide. However, they are essentially different within the near zone.

Figure 3 compares the nonasymptotic cross section $d \sigma(\eta)$, the Mott cross section $d \sigma_{M}(\eta)$, and the Rutherford cross section $d \sigma_{\mathrm{NR}}(\eta)$ for some values of the scattering parameters. One can see that the value $d \sigma(\eta)$ is finite and coincides with $d \sigma_{M}(\eta)$ when $\eta \gg 1, \theta \gg \theta_{0}$. Besides, $d \sigma(\eta)$ converges to the result from Ref. [6] for a nonrelativistic electron $\left(p_{0}<m\right)$ when $d \sigma_{M}(\eta) \rightarrow d \sigma_{\mathrm{NR}}(\eta)$. It should be noticed that the scale along the $\eta$ axis is proportional to the value $\sqrt{p_{0}}$.

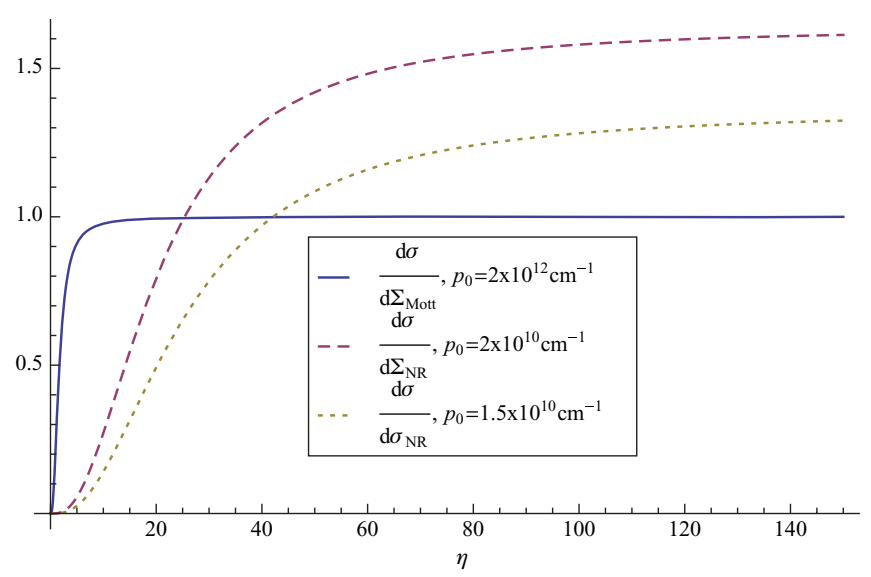

FIG. 3. (Color online) Comparison between the nonasymptotic cross section $d \sigma$, the Mott cross section $d \sigma_{M}$ for the electrons with various momenta $p_{0}$, and the Rutherford cross section $d \sigma_{\mathrm{NR}}$; atomic number $Z=80$; the distance from the scattering center is $r=100 \mathrm{~cm} ; \tilde{\delta}=3$; and $m \approx 2.5 \times 10^{10} \mathrm{~cm}^{-1}$ in the considered system of units. 


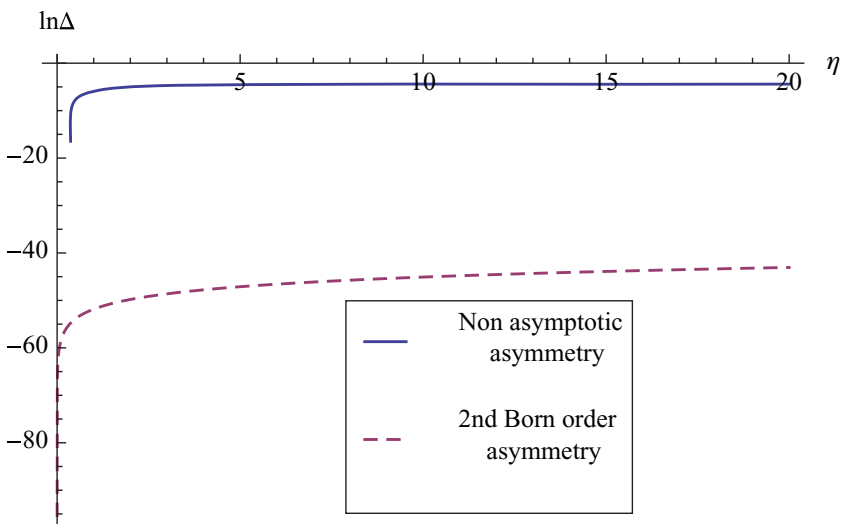

FIG. 4. (Color online) Comparison of the nonasymptotic asymmetry with the second-order Born asymmetry for momentum $p_{0}=$ $2 \times 10^{12} \mathrm{~cm}^{-1}$, atomic number $Z=80$, and a distance from the scattering center of $r=100 \mathrm{~cm}, \tilde{\delta}=3$. Logarithmic scale has been used.

The asymmetry of the scattering in a small angular region is calculated as follows:

$$
\begin{gathered}
\Delta(\eta)=\frac{\tilde{g}}{\tilde{f}}, \\
\tilde{g}=|C|^{2} \frac{4 \pi r}{p_{0}} \eta \xi \frac{m}{\varepsilon_{0}} \\
\times \operatorname{Re}\left[\frac{U_{1}\left(i \xi, 1, i \eta^{2}\right) U_{1}^{*}\left(i \xi+1,2, i\left(\eta^{2}+\frac{\tilde{\delta}^{2}}{2}\right)\right)}{\Gamma(i \xi) \Gamma^{*}(i \xi+1)}\right] .
\end{gathered}
$$

This value can be compared with the result of the Born series to second order [2] written in the same variables:

$$
\Delta(\eta)=\xi \frac{v_{0}^{2} \sqrt{1-v_{0}^{2}}}{\left(2 p_{0} r\right)^{3 / 2}} \eta^{3} \ln \left(\frac{\eta^{2}}{2 p_{0} r}\right) .
$$

Figure 4 represents this comparison in logarithmic scale because the asymmetry is a small value.

As was stated above, the asymmetry depends on $\tilde{\delta}$ as the "average packet parameter." Figure 5 shows the asymmetry for different values of $\tilde{\delta}$.

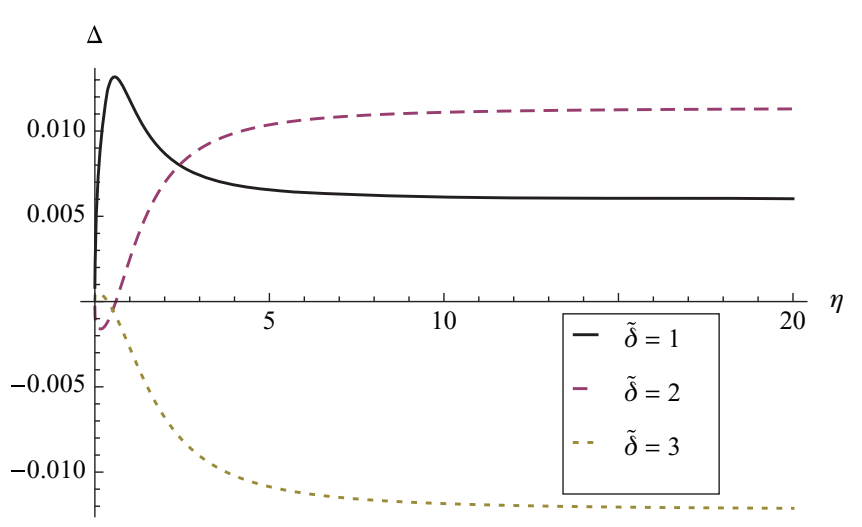

FIG. 5. (Color online) The asymmetry dependence on $\tilde{\delta}$ for momentum $p_{0}=2 \times 10^{12} \mathrm{~cm}^{-1}$, atomic number $Z=80$, and a distance from the scattering center of $r=100 \mathrm{~cm}$.

\section{NUMERICAL RESULTS FOR THE INTEGRAL SCATTERING CHARACTERISTICS}

Let us now compare numerically the nonasymptotic scattering theory considered above with the results of the conventional theory for relativistic particle scattering in a Coulomb field. The differential cross section proved to be an integrable function, which leads to the possibility of calculating the total scattering cross section. This integral characteristic becomes a function of the distance $r$ between the scattering center and observation point. We consider the case when the initial electron beam is not polarized. The problem comes to calculating the following integral:

$$
\sigma_{\mathrm{tot}}=\int_{0}^{\pi} 2 \pi|C|^{2} s \sin \theta d \theta
$$

where

$$
s=\frac{d \sigma^{(1)}}{d \Omega}+\frac{d \sigma^{(2)}}{d \Omega}+\frac{d \sigma^{(4)}}{d \Omega} .
$$

Let us insert a new variable $z_{0}$

$$
\begin{aligned}
& z_{0}=\operatorname{pr}(1-\cos \theta), \quad \sin \theta d \theta=\frac{d z_{0}}{p_{0} r}, \\
& \cos \theta=1-\frac{z_{0}}{p_{0} r}, \quad \sin ^{2} \theta / 2=\frac{z_{0}}{2 p_{0} r},
\end{aligned}
$$

in this integral:

$$
\begin{aligned}
\sigma_{\text {tot }}= & \int_{0}^{2 p_{0} r} \frac{2 \pi r}{p_{0}} \frac{|C|^{2}}{|\Gamma(i \xi)|^{2}}\left|U_{1}\left(i \xi, 1, i z_{0}\right)\right|^{2} d z_{0} \\
& -\int_{0}^{2 p_{0} r}\left(\frac{2 \pi}{p_{0}^{2}} \frac{|C|^{2}}{|\Gamma(i \xi)|^{2}}\left|U_{1}\left(i \xi, 1, i z_{0}\right)\right|^{2}\right. \\
& +\frac{2 \pi \xi}{p_{0}^{2}}\left\{K_{1} \operatorname{Im}\left[U_{1}\left(i \xi, 1, i z_{0}\right) U_{1}^{*}\left(i \xi+1,2, i z_{0}\right)\right]\right. \\
& \left.+K_{2} \operatorname{Re}\left[U_{1}\left(i \xi, 1, i z_{0}\right) U_{1}^{*}\left(i \xi+1,2, i z_{0}\right)\right]\right\}|C|^{2}-\frac{\pi \xi^{2} v_{0}^{2}}{p_{0}^{2}} \\
& \left.\times \frac{|C|^{2}}{|\Gamma(i \xi+1)|^{2}}\left|U_{1}\left(i \xi+1,2, i\left(z_{0}+\frac{\tilde{\delta}^{2}}{2}\right)\right)\right|^{2}\right) z_{0} d z_{0},
\end{aligned}
$$

where

$K_{1}=\operatorname{Re}\left(\frac{1}{\Gamma(i \xi) \Gamma^{*}(i \xi+1)}\right), K_{2}=\operatorname{Im}\left(\frac{1}{\Gamma(i \xi) \Gamma^{*}(i \xi+1)}\right)$.

Let us now analyze the dependence of each integral in Eq. (66) on the distance $r$. To calculate these integrals let us express each of them in terms of other integrals. For example,

$$
\begin{aligned}
i_{1}(\xi)= & \int_{0}^{2 p_{0} r}\left|U_{1}\left(i \xi, 1, i z_{0}\right)\right|^{2} d z_{0}=\int_{0}^{\infty}\left|U_{1}\left(i \xi, 1, i z_{0}\right)\right|^{2} d z_{0} \\
& -\int_{2 p_{0} r}^{\infty}\left|U_{1}\left(i \xi, 1, i z_{0}\right)\right|^{2} d z_{0} .
\end{aligned}
$$

In the second integral, the asymptotic representation of $U_{1}(a, b, z)$,

$$
U_{1}(a, b, z)=|z|^{a-b} e^{i \arg (z)(a-b)} e^{z}
$$


can be used:

$$
\int_{2 p_{0} r}^{\infty}\left|U_{1}\left(i \xi, 1, i z_{0}\right)\right|^{2} d z_{0}=e^{-\pi \xi} \int_{2 p_{0} r}^{\infty} \frac{d z_{0}}{z_{0}^{2}}=\frac{e^{-\pi \xi}}{2 p_{0} r} .
$$

This means that this contribution can be neglected in Eq. (66) since $\sim\left(p_{0} r\right)^{-1} \ll 1$. Hence,

$$
i_{1}(\xi)=\int_{0}^{\infty}\left|U_{1}\left(i \xi, 1, i z_{0}\right)\right|^{2} d z_{0}
$$

and does not depend on $r$.

The next integral,

$$
\int_{0}^{2 p_{0} r}\left|U_{1}\left(i \xi, 1, i z_{0}\right)\right|^{2} z_{0} d z_{0}
$$

can be also represented identically as a sum of converged integrals, but in a different way:

$$
\begin{aligned}
\int_{0}^{2 p_{0} r}\left|U_{1}(i \xi, 1, i z)\right|^{2} z_{0} d z_{0} \\
=\int_{0}^{1}\left|U_{1}\left(i \xi, 1, i z_{0}\right)\right|^{2} z_{0} d z_{0}+\int_{1}^{2 p_{0} r}\left|U_{1}\left(i \xi, 1, i z_{0}\right)\right|^{2} z_{0} d z_{0} \\
=\int_{0}^{1}\left|U_{1}\left(i \xi, 1, i z_{0}\right)\right|^{2} z_{0} d z_{0}+\int_{1}^{2 p_{0} r}\left(\left|U_{1}\left(i \xi, 1, i z_{0}\right)\right|^{2}\right. \\
\left.\quad-\frac{e^{-\pi \xi}}{z_{0}^{2}}\right) z_{0} d z_{0}+\int_{1}^{2 p_{0} r} \frac{e^{-\pi \xi}}{z_{0}^{2}} z_{0} d z_{0} .
\end{aligned}
$$

In the second term of Eq. (70), the upper limit of integration can be replaced by infinity, because of the asymptotic form (68) of the $U_{1}(i \xi, 1, i z)$ function. The intermediate integration point has been chosen for regularization of the asymptotic part of the integral. Then the last term of Eq. (70) can be calculated analytically:

$$
\begin{aligned}
& \int_{0}^{2 p_{0} r}\left|U_{1}\left(i \xi, 1, i z_{0}\right)\right|^{2} z_{0} d z_{0} \\
& =\int_{0}^{1}\left|U_{1}\left(i \xi, 1, i z_{0}\right)\right|^{2} z_{0} d z_{0} \\
& \quad+\int_{1}^{\infty}\left(\left|U_{1}\left(i \xi, 1, i z_{0}\right)\right|^{2}-\frac{e^{-\pi \xi}}{z_{0}^{2}}\right) z_{0} d z_{0}+e^{-\pi \xi} \ln 2 p_{0} r \\
& =i_{2}(\xi)+e^{-\pi \xi} \ln 2 p_{0} r,
\end{aligned}
$$

with integral $i_{2}(\xi)$ that does not depend on $r$.

The same procedure can be followed for the other integrals:

$$
\begin{aligned}
& \int_{0}^{2 p_{0} r} \operatorname{Re}\left(U_{1}\left(i \xi, 1, i z_{0}\right) U_{1}^{*}\left(i \xi+1,2, i z_{0}\right)\right) z_{0} d z_{0} \\
& =\int_{0}^{1} \operatorname{Re}\left(U_{1}\left(i \xi, 1, i z_{0}\right) U_{1}^{*}\left(i \xi+1,2, i z_{0}\right)\right) z_{0} d z_{0} \\
& \quad+\int_{1}^{\infty}\left[\operatorname{Re}\left(U_{1}\left(i \xi, 1, i z_{0}\right) U_{1}^{*}\left(i \xi+1,2, i z_{0}\right)\right)-\frac{e^{-\pi \xi}}{z_{0}^{2}}\right] \\
& \quad+e^{-\pi \xi} \ln 2 p_{0} r=i_{3}(\xi)+e^{-\pi \xi} \ln 2 p_{0} r
\end{aligned}
$$

and

$$
\begin{aligned}
\int_{0}^{2 p_{0} r} \operatorname{Im}\left(U_{1}\left(i \xi, 1, i z_{0}\right) U_{1}^{*}\left(i \xi+1,2, i z_{0}\right)\right) z_{0} d z_{0} \\
\quad=\int_{0}^{\infty} \operatorname{Im}\left(U_{1}\left(i \xi, 1, i z_{0}\right) U_{1}^{*}\left(i \xi+1,2, i z_{0}\right)\right) z_{0} d z_{0}=i_{4}(\xi) .
\end{aligned}
$$

The last integral that remains on the right-hand side of Eq. (66) consists of $\tilde{\delta}$. However, when one goes to infinity, the asymptotic form happens not to depend on $\tilde{\delta}$, as it should be. That is why the last integral can be calculated in the same way as Eqs. (71)-(73),

$$
\begin{aligned}
& \int_{0}^{2 p_{0} r}\left|U_{1}\left(i \xi+1,2, i\left(z_{0}+\frac{\tilde{\delta}^{2}}{2}\right)\right)\right|^{2} z_{0} d z_{0} \\
& =\int_{0}^{1}\left|U_{1} i\left(\xi+1,2, i\left(z_{0}+\frac{\tilde{\delta}^{2}}{2}\right)\right)\right|^{2} z_{0} d z_{0} \\
& +\int_{1}^{\infty}\left(\left|U_{1}\left(i \xi+1,2, i\left(z_{0}+\frac{\tilde{\delta}^{2}}{2}\right)\right)\right|^{2}-\frac{e^{-\pi \xi}}{z_{0}^{2}}\right) z_{0} d z_{0} \\
& \quad+\int_{1}^{2 p_{0} r} \frac{e^{-\pi \xi}}{z_{0}^{2}} z_{0} d z_{0}=i_{5}(\xi)+e^{-\pi \xi} \ln 2 p_{0} r
\end{aligned}
$$

with integral $i_{5}(\xi)$ that is the function of the wave-packet parameter $\tilde{\delta}$ but does not depend on $r$.

Substituting Eqs. (67)-(74) into Eq. (66), one can obtain a finite value for the total cross section of the electron scattering by the Coulomb center:

$$
\begin{aligned}
\sigma_{\text {tot }}= & \frac{2 \pi r}{p_{0}} \frac{|C|^{2}}{|\Gamma(i \xi)|^{2}} i_{1}(\xi)-\frac{2 \pi}{p_{0}^{2}} \frac{|C|^{2}}{|\Gamma(i \xi)|^{2}} i_{2}(\xi) \\
& +|C|^{2} \frac{2 \pi \xi}{p_{0}^{2}}\left(K_{1} i_{4}(\xi)+K_{2} i_{3}(\xi)\right) \\
& -\frac{\pi \xi^{2} v_{0}^{2}}{p_{0}^{2}} \frac{|C|^{2}}{|\Gamma(i \xi+1)|^{2}} i_{5}(\xi)
\end{aligned}
$$
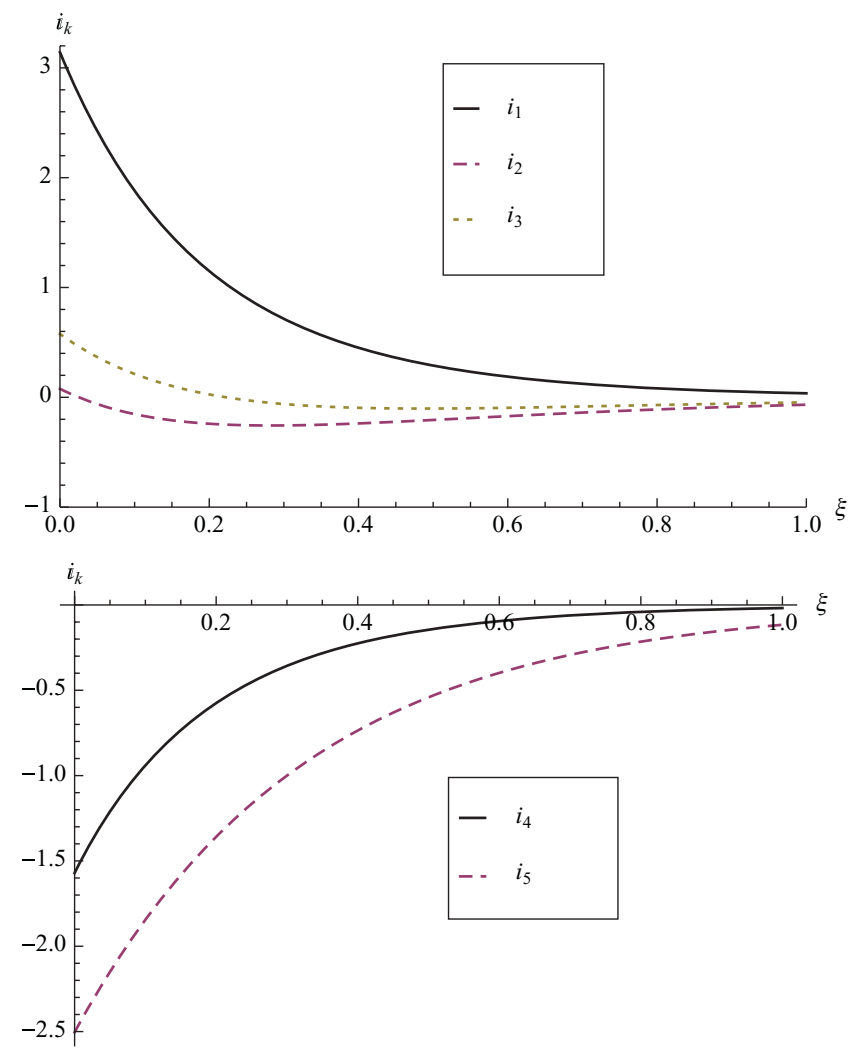

FIG. 6. (Color online) Universal functions $i_{k}(\xi), k=\overline{1, \ldots, 5}$, which define the total cross section as a function of $\xi$. 


$$
\begin{aligned}
& +\frac{2 \pi|C|^{2}}{p_{0}^{2}} e^{-\pi \xi} \ln 2 p_{0} r\left(\xi K_{2}-\frac{1}{|\Gamma(i \xi)|^{2}}\right. \\
& \left.-\frac{\xi^{2} v_{0}^{2}}{2} \frac{1}{|\Gamma(i \xi+1)|^{2}}\right) .
\end{aligned}
$$

The universal functions $i_{k}(\xi), k=\overline{1, \ldots, 5}$ depend on only one parameter $\xi$ and are defined by convergent integrals, which can be easily calculated numerically. Figure 6 shows the results. It should be noted that the dependence of the integral $i_{5}(\xi)$ on the parameter $1<\tilde{\delta}<5$ is invisible in the scale used.

It should be noted that the value $r$ is restricted because of inequality (9), and the maximum value for the total cross section is

$$
\sigma_{\text {tot }}^{\max }=2 \pi a^{2} \frac{p_{0}^{2}}{m^{2}} f(\xi), \quad f(\xi) \leqslant 1,
$$

which can be compared with $\sigma_{\text {tot }}^{\max }=2 \pi a^{2} f_{1}(\xi)$ in the nonrelativistic case [6].

\section{CONCLUSIONS}

In this paper we have studied the question whether the Coulomb singularity of the Mott cross section is an intrinsic feature of the Coulomb potential or if one could avoid it on the basis of a nonasymptotic calculation. Important peculiarities have been discussed for electron scattering in the Coulomb

[1] L. D. Landau and E. M. Lifshitz, Quantum Mechanics: Nonrelativistic Theory, 3rd ed. (Pergamon, London, 1997), Vol. 3.

[2] A. I. Akhiezer and V. B. Berestetsky, Quantum Electrodynamics (Nauka, Moscow, 1969).

[3] E. Conwell and V. F. Weisskopf, Phys. Rev. 77, 388 (1950).

[4] H. Brooks, Phys. Rev. 83, 879 (1951).

[5] N. A. Poklonski et al., Phys. Status Solidi B 244, 3703 (2007).

[6] V. G. Baryshevskii, I. D. Feranchuk, and P. B. Kats, Phys. Rev. A 70, 052701 (2004).

[7] V. G. Baryshevskii, L. I. Korennaya, and I. D. Feranchuk, Sov. Phys. JETP 34, 249 (1972). field: In the general case, the scattering characteristics depend on the observation point coordinate $r$ and on the wave-packet parameter. These peculiarities are due to the long-range character of the Coulomb potential and should be analyzed in a nonasymptotic way analogously to the nonrelativistic case [6]. Nonasymptotic effects are essential only in the small-angle range

$$
\theta<\theta_{0}=\sqrt{\frac{2}{p_{0} r}},
$$

where the plane and spherical waves cannot be distinguished.

In spite of the small value of $\theta_{0}$, these effects lead to a finite contribution to the total cross section and change the scattered particle's polarization. These values prove to be well-defined functions of $r$. It is important to emphasize that the considered peculiarities of the Coulomb problem should be taken into account only for the potential without any screening.

\section{ACKNOWLEDGMENTS}

The authors are grateful to Professor V. G. Baryshevsky for useful discussions. We are also grateful to Professor H. Kleinert for the interest shown in this work and to the Center of International Cooperation of the Free University of Berlin for financial support.

[8] W. H. Furry, Phys. Rev. 46, 391 (1934).

[9] M. J. Roberts, J. Phys. B 18, L707 (1985).

[10] J. Macek, Phys. Rev. A 37, 2365 (1988).

[11] J. Botero and J. H. Macek, Phys. Rev. A 45, 154 (1992).

[12] G. Gasaneo and L. U. Ancarani, Phys. Rev. A 80, 062717 (2009).

[13] M. L. Goldberger and K. M. Watson, Collision Theory (Wiley, New York, 1964).

[14] P. M. Morse and H. Feshbach, Methods of Theoretical Physics (McGraw-Hill, New York, 1953). 